\title{
A Numerical Study of Topological Features of Certain Hilbert Fundamental Domains*
}

\author{
By Harvey Cohn
}

9. Revision of Program. An earlier program, described in [6], tells how we can examine the three-dimensional floor of a four-dimensional Hilbert fundamental domain. The geometry of such domains is of recent analytic interest [2], [8], but we had done little more than count the number of essentially different three-dimensional "analytic pieces." Actually, these pieces paired off under the modular group $H$ to form an "essentially compact" domain just as the opposite sides of the well-known period parallelogram pair off under their translation group to form a torus.

We revise this earlier program so as to clarify the pairing process and the relative positions of these three-dimensional analytic pieces of the floor. We restrict ourselves to the fields of $k^{1 / 2}$ where $k=5,2,3,6$; indeed, even these cases reveal the practical limitations of our methods. The fact that we are pairing off points in an interesting fibre space (see Section 12 below) might justify the crude efforts of the present attempt at visualization.

To understand the desired revision, let us recall that (as in (3.16)) the last program provided us with output consisting of

$$
Z, Z^{\prime} ; \gamma, \gamma^{\prime} ; \delta, \delta^{\prime}
$$

where $Z, Z^{\prime}$ is a point of the floor on the analytical three-dimensional piece provided by

$$
\|\gamma Z+\delta\|=|\gamma Z+\delta|^{2}\left|\gamma^{\prime} Z^{\prime}+\delta^{\prime}\right|^{2}=1 .
$$

What we want to do is to interlace each print-out (9.1) with

$$
Z_{*}, Z_{*}{ }^{\prime} ; \alpha, \alpha^{\prime} ; \beta, \beta^{\prime}
$$

where $Z_{*}, Z_{*}{ }^{\prime}$ represents the matching point paired with $Z, Z^{\prime}$ by

$$
Z_{*}=(\alpha Z+\beta) /(\gamma Z+\delta)
$$

(and likewise for the "primed" quantities). Here $\alpha, \beta \in \boldsymbol{O}$ (just as $\gamma, \delta$ ) and

$$
\alpha \delta-\beta \gamma=\epsilon(\gg 0)
$$

for $\epsilon$ a unit in $\boldsymbol{O}$.

In the more recent paper [7], it is shown that exactly one $Z_{*}, Z_{*}{ }^{\prime}$ (of the floor) pairs off with each $Z, Z^{\prime}$ (of the floor) under $H$ except for sets of lower dimension

Received March 21, 1966.

* Research supported by NSF Grant G-7412. Computer assistance furnished through the cooperation of Dr. Wallace J. Givens, Director, and Mr. Burton S. Garbow, Programmer, Applied Mathematics Division, Argonne National Laboratory of the U.S.A.E.C. The sections, remarks, bibliographical items, etc., are numbered consecutively with the previous paper [6] (see bibliography below). The symbols are defined in Section 2 or Section 3 of [6] unless other references are given. 
than three, where several different pairings can occur. (This is like saying that two opposite sides of the period parallelogram pair off "pointwise" uniquely except for each of four corners which pairs off with any other corner.)

Actually, the print-out (9.3) appears in a fashion very similar to (3.16), except for the omission of the "epsilon" (error $S_{2}$ ):

$$
R_{1}^{*}, R_{2}^{*}, S_{1}^{*}, S_{2}^{*}, X_{*}, X_{*}^{\prime}, Y_{*}, Y_{*}^{\prime}, a_{1}, a_{2}, b_{1}, b_{2} \text {. }
$$

Here, in keeping with the usual terminology of $(2.2)$, we set

$$
Z_{*}=X_{*}+i Y_{*}, \quad Z_{*}{ }^{\prime}=X_{*}^{\prime}+i Y_{*}^{\prime}
$$

and we define $R_{1}{ }^{*}, R_{2}{ }^{*}$ by (2.9) and $S_{1}{ }^{*}, S_{2}{ }^{*}$ by (3.1) and (3.2). Finally, analogously with (2.1) or

$$
\gamma=g_{1}+g_{2} \omega, \quad \delta=d_{1}+d_{2} \omega
$$

we set

$$
\alpha=a_{1}+a_{2} \omega, \quad \beta=b_{1}+b_{2} \omega .
$$

Thus, we want to obtain successive pairs (3.16) and (9.6) (schematically representing (9.1) and (9.3)) from the revised computation.

10. Computation Procedure. The denominator in (9.4) was seen (in Section 3 ) to be determined by the $\gamma, \delta$ of (9.2). Hence, only $\alpha, \beta$ need be determined. Thus, from (9.1) we set up a "first approximation" to $Z_{*}$, namely

$$
Z_{0}=\left(\alpha_{0} Z+\beta_{0}\right) /(\gamma Z+\delta)
$$

where $\alpha_{0}$ and $\beta_{0}$ are determined in $O$ so as to make $\alpha_{0} \delta-\beta_{0} \gamma=1$ (the most convenient totally-positive unit).

(i) Therefore, the earlier runs are scanned and the output values of $(\gamma, \delta)$ (actually $g_{1}, g_{2}, d_{1}, d_{2}$ ) are listed manually with arbitrary values of $\alpha_{0}, \beta_{0}$ satisfying $\alpha_{0} \delta-\beta_{0} \gamma=1$ (as calculated manually). This creates an 8 column table of integers if we set $\alpha_{0}=a_{1}{ }^{0}+\omega a_{2}{ }^{0}, \beta_{0}=b_{1}{ }^{0}+\omega b_{2}{ }^{0}$ with each line looking like

$$
g_{1}, g_{2}, d_{1}, d_{2} ; \quad a_{1}^{0}, a_{2}^{0}, b_{1}^{0}, b_{2}^{0} \text {. }
$$

(See Remark (e) in Section 13 below.) The machine can now look up $\alpha_{0}$ and $\beta_{0}$ for each $\gamma, \delta$.

Thus from any set of data (9.1) the machine finds $\alpha_{0}$ and $\beta_{0}$ from the table (10.2). Such values are not unique, to the extent that

$$
Z_{*}=\epsilon_{+}{ }^{t} Z_{0}+\nu
$$

where the integer $t$ and $\nu$ remain to be determined. The machine determines $\epsilon_{+}{ }^{t}$ by asking if the ratio $Y_{0}^{\prime} / Y_{0}=S_{1}^{0}$ (formed from $Z_{0}, Z_{0}^{\prime}$ ) satisfies

$$
h \geqq S_{1}{ }^{0}>-h
$$

as required by the fundamental domain (see (3.3)). Therefore according as $S_{0}$ is too big $(>h)$ or too small $(<-h)$, we replace $\left(Z_{*}, Z_{*}{ }^{\prime}\right)$ by $\left(\epsilon_{+} Z_{*}, \epsilon_{+}{ }^{-1} Z_{*}{ }^{\prime}\right)$ or by $\left(\epsilon_{+}^{-1} Z_{*}, \epsilon_{+} Z_{*}{ }^{\prime}\right)$. This process is "looped" until (10.4) is satisfied by (the new) $S_{1}{ }^{0}$ (see Remark (f)). 
(ii) Therefore the further input information is required, consisting of the ratios (see Table 1, Section 3 )

$$
h=h_{1} / h_{2}
$$

written as an actual fraction (since $h_{2}$ would not always be 1 if the range of $k$ is extended) and consisting of the fundamental totally-positive unit

$$
u_{1}, u_{2} \quad\left(\epsilon_{+}=u_{1}+u_{2} \omega\right) .
$$

Now we have a correct value of $\epsilon_{+}{ }^{t} Z_{0}$, but we need to add $\nu$ to it to bring it under the translational limitations $(2.9 \mathrm{a}, \mathrm{b})$. Here we convert $\epsilon_{+}{ }^{t} Z_{0}, \epsilon_{+}{ }^{-t} Z_{0}^{\prime}$ into the $R_{1}, R_{2}, S_{1}, S_{2}$ values. We find $S_{1}$ within (10.4) but $R_{1}$ and $R_{2}$ do not yet lie within $\left(-\frac{1}{2}, \frac{1}{2}\right)$. We therefore let $M_{i}$ be the closest integer to $R_{i}(i=1,2)$ and set

$$
-\nu=M_{1}+M_{2} \omega,-\nu^{\prime}=M_{1}+M_{2} \omega^{\prime}
$$

and finally obtain $Z_{*}=\epsilon_{+}{ }^{t} Z_{0}+\nu$, etc., in the form $R_{1}{ }^{*}, R_{2}{ }^{*}, S_{1}{ }^{*}, S_{2}{ }^{*}$.

As an added check on accuracy, $S_{2}=S_{2}{ }^{*}$, ( since each of these two values is supposed to be a maximum under linear transformations (9.4)). We achieve this to the print-out accuracy (of $10^{-4}$ ) with the error limit of $10^{-6}$ in solving (3.11). It should be clear to the reader how the information in (i) and (ii) is entered into the memory by additional input cards.

11. Interpretation of Invariants. We are now in a position to see how the analytic pieces of the floor will pair off, but we must recall that the meaning of "pieces" is somewhat artificial. We insist on the sanctity of the "wedge relationship" (2.8) or (3.3) but we permit the translation operations $Z \rightarrow Z+\nu$ to be freely used so as to sacrifice the parallelogram relations (2.9a) in the idea of an "analytic piece." In other words, each piece is identified only by the invariant residue class of $\delta$ modulo $\gamma$, for which we say the center is at

$$
x=-\delta / \gamma, \quad x^{\prime}=-\delta^{\prime} / \gamma^{\prime} \text {. }
$$

The classes are listed in Table 3 (of Section 7 above).

In the case of a simple floor, $k=5$, actually 7 "subpieces" are translated to form the single "analytic piece" (see Section 7 above). These "subpieces" all belong to the residue class of $0(\bmod 1)$. Their interrelation is too complicated to draw even from what we conjecture to be a completely adequate sample of points. Hence no diagram is offered for $k=5$ and in any other case the "piece" (or "subpieces") corresponding to $0(\bmod 1)$ will be ignored.

Generally speaking the transformation $(9.4)$ can be written as

$$
\left(\gamma Z_{*}-\alpha\right)=-\epsilon /(\gamma Z+\delta)
$$

Hence the piece which pairs off with " $\delta$ modulo $\gamma$ " is simply " $-\alpha$ modulo $\gamma$ " where

$$
\alpha \delta \equiv \epsilon(\bmod \gamma) \text {. }
$$

The center (11.1) becomes matched with the center

$$
x_{*}=\alpha / \gamma, \quad x_{*}{ }^{\prime}=\alpha^{\prime} / \gamma^{\prime} .
$$

Furthermore, if we introduce the new variables (actually conjugate pairs)

$$
\zeta=\gamma Z+\delta, \quad \zeta^{*}=\gamma Z_{*}-\alpha
$$


the transformations take the convenient form

$$
\zeta \zeta^{*}=-\epsilon
$$

which can be explained as an "approximate" inversion relation about the centers (11.1) and (11.4), (remember that $S_{2}$ and $Y, Y^{\prime}, Y_{*}, Y_{*}{ }^{\prime}$ are all bounded from below so that imaginary parts never vanish).

An examination of the output leads to Table 4 for the cases $k=2,3,6$ and $|N(\gamma)|>1$. The labeling of the centers by subscripts showing the norm should be self-explanatory. We note that the centers (11.1) and (11.4) are fortuitously the same $(\alpha+\delta=0)$ so only one need be listed. The pieces also will be labelled for convenience by $|N(\gamma)|$ except when $k=6$, we use 3 and $3^{\prime}$ to denote the two pieces of norm 3.

In the case $k=2, \gamma=2^{1 / 2}$, the designation $\zeta_{2}=-1 / \zeta_{2}{ }^{*}$ stands for four different transformations in the output, namely $Z=T\left(Z^{*}\right)$ where

$$
\begin{array}{r}
T\left(Z^{*}\right)=Z^{*} /\left(2^{1 / 2} Z^{*}+1\right),-Z^{*} /\left(2^{1 / 2} Z^{*}-1\right),\left(-Z^{*}-2^{1 / 2}\right) /\left(2^{1 / 2} Z^{*}+1\right), \\
\text { and }\left(Z^{*}-2^{1 / 2}\right) /\left(2^{1 / 2} Z^{*}-1\right) .
\end{array}
$$

For the case $k=3, \gamma=1+3^{1 / 2}$, the number of transformations involved is 16 . This should give the reader an idea of the number of different matchings of the subpieces of just one analytic piece!

12. Visualization of Pieces of Floor. Let us now reconsider the mode of representation of the floor. It consists of a hypersurface of dimension 3 taking the form $S_{2}=f\left(R_{1}, R_{2}, S_{1}\right)$ (see (3.15)), but the domain of $R_{1}, R_{2}, S_{1}$ is a rectangular parallelopiped with "torus-like" identifications of boundaries. Indeed, from (2.9a), $R_{1}, R_{2}$ constitutes a 2 -torus (taking each coordinate modulo 1 ); and these 2 -tori are fibres while $S_{1}$ varies (see (3.3)) over the range $(-h, h)$ constituting the base of a fibre space (see [10]). The periodicity of $S_{1}$ (modulo $2 h$ ) makes the base a onesphere (or one-torus for that matter) but a group is associated with the fibre space. If $\left(R_{1}^{+}, R_{2}^{+}\right)$denotes the torus at $S_{1}=+h$ and $\left(R_{1}^{-}, R_{2}^{-}\right)$denotes the torus at $S_{2}=-h$ then the tori are defined by $Z^{-}=Z^{+} \epsilon_{+}$or

$$
\left(R_{1}^{+}+k^{1 / 2} R_{2}^{+}\right) \epsilon_{+} \equiv\left(R_{1}^{-}+k^{1 / 2} R_{2}^{-}\right) \quad(\bmod O) .
$$

TABLE 4

Matching Relations of Pieces in Table 3 for $|N(\gamma)|>1$

\begin{tabular}{l|c|c|c|c|c}
\hline$k$ & $\begin{array}{c}|N(\gamma)| \\
(\text { label })\end{array}$ & $\gamma$ & $\begin{array}{c}-\delta, \alpha \\
(\bmod \gamma)\end{array}$ & $\begin{array}{c}\epsilon \\
(\mathrm{Eq} . \\
(11.6))\end{array}$ & $\begin{array}{c}x_{|N(\gamma)|} \\
(\mathrm{Eq} \cdot(11.1))\end{array}$ \\
\hline 2 & 2 & $\begin{array}{c}2^{1 / 2} \\
1+3^{1 / 2}\end{array}$ & 1,1 & 1 & $-2^{1 / 2} / 2$ \\
3 & 2 & 1,1 & $\epsilon_{+}$ & $\left(-1-3^{1 / 2}\right) / 2$ \\
6 & 2 & $2+6^{1 / 2}$ & 1,1 & $\epsilon_{+}$ & $-6^{1 / 2} / 2$ \\
& 3 & $3+6^{1 / 2}$ & 1,1 & $\epsilon_{+}^{1 / 2} / 3$ \\
& $3+6^{1 / 2}$ & $-1,-1$ & $\epsilon_{+}$ & $6^{1 / 2} / 3$ \\
& 4 & 2 & $1+6^{1 / 2}, 1+6^{1 / 2}$ & 1 & $\left(-1-6^{1 / 2}\right) / 2$ \\
\hline
\end{tabular}


Here we exclude $k \equiv 1(\bmod 4)$ for convenience and recall that $\epsilon_{+}$is written as $u_{1}+u_{2} k^{1 / 2}$. Then (12.1) is a matrix relation (modulo one)

$$
M_{k}\left(\begin{array}{l}
R_{1}^{+} \\
R_{2}^{+}
\end{array}\right)=\left(\begin{array}{l}
R_{1}^{-} \\
R_{2}^{-}
\end{array}\right) ; \quad M_{k}=\left(\begin{array}{ll}
u_{1} & k u_{2} \\
u_{2} & u_{1}
\end{array}\right) ;
$$

where a fairly complicated pattern of identifications emerges for the tori at $S_{1}=+h$ and $-h$, e.g.,

$$
M_{2}=\left(\begin{array}{ll}
3 & 4 \\
2 & 3
\end{array}\right), \quad M_{3}=\left(\begin{array}{ll}
2 & 3 \\
1 & 2
\end{array}\right), \quad M_{6}=\left(\begin{array}{ll}
5 & 12 \\
2 & 5
\end{array}\right) .
$$

Thus we are dealing with the fibre space of a 3-torus under group (12.2) with further interior identifications in accordance with (9.1) and (9.3). The homology group of the fibre space is determined by the vertical sections; i.e., the lines $R_{1}=$ const, $R_{2}=$ const, determined by

$$
M_{k}\left(\begin{array}{l}
R_{1} \\
R_{2}
\end{array}\right) \equiv\left(\begin{array}{l}
R_{1} \\
R_{2}
\end{array}\right) \quad(\bmod 1) .
$$

The matter of these homology groups is discussed further in [9], but right here, the important fact is that an analytic piece of the floor often extends from $S_{1}=-h$ to $S_{1}=+h$ where it is pinched to the same point $R_{1}=$ const, $R_{2}=$ const at both end points. This can happen only when $\left(R_{1}, R_{2}\right)$ corresponds to a vertical section. Examples of this occur in each case.

$k=2$. When $k=2$, the piece of norm 2 is very much like an ordinary propeller (airscrew) extending along to vertical section at $x_{2}$ or $R_{1}=0, R_{2}=-\frac{1}{2}$ and pinched to a point not only at $S_{1}= \pm 1$ but also at $S_{1}=0$. We display some $\left(R_{1}, R_{2}\right)$ cross-sections in Fig. 2 so as to convey the idea of a "twisted propeller blade."

We have a final adaptation of the output where the norm of each point of the floor is printed (as a single digit) at the lattice points giving an immediate view of the pieces in the $\left(R_{1}, R_{2}\right)$ cross-section for constant $S_{1}(<0)$. The diagrams are self-explanatory. In each case, the symmetry $R_{1} \rightarrow+R_{1}, R_{2} \rightarrow-R_{2}, S_{1} \rightarrow-S_{1}$ (a reflection about $R_{1}=-\frac{1}{2}$ in the $\left(R_{1}, R_{2}\right)$ plane), will enable us to imagine the rest of the crosssections, where $S_{1}>0$.

$k=3$. We do likewise for $k=3$ displaying cross-section again, but here the piece of norm 2 becomes pinched to a point only at $S_{1}= \pm 1$ and not at $S_{1}=0$. When $S_{1}=0$, this piece bulges around $x_{2}$ at $\left(R_{1}, R_{2}\right)=\left(-\frac{1}{2},-\frac{1}{2}\right)$ so as to reach across from $\left(R_{1}, R_{2}\right)=\left(-1,-\frac{1}{2}\right)$ to $\left(0,-\frac{1}{2}\right)$, as shown in Fig. 3 . (Here we always use coordinates $R_{1}$ and $R_{2}$ modulo 1 .)

In both Fig. 2 and Fig. 3 the center $x_{2}$ lines at the center of each cross-section.

$k=6$. For $k=6$ we have a much more complicated situation. The piece of the floor of norm 4 is again propeller-shaped, surrounding the vertical section at $x_{4}$ of $\left(R_{1}, R_{2}\right)=\left(-\frac{1}{2},-\frac{1}{2}\right)$ and pinched to a point at $S_{1}=0, \pm 2$ (similar to the piece of norm 2 for $k=2$ ).

The piece of norm 3 is, however, like a "bent" propeller. It starts at $x_{4}$ or $\left(R_{1}\right.$, $\left.R_{2}\right)=\left(-\frac{1}{2},-\frac{1}{2}\right)$ for $S_{1}=-2$ and extends to $x_{3}$ at $\left(0,-\frac{1}{3}\right)$ at $S_{1}=0$ where it is pinched to a point. It continues from $x_{3}$ or $\left(0,-\frac{1}{3}\right) \equiv\left(-1,-\frac{1}{3}\right)$ back to $\left(-\frac{1}{2},-\frac{1}{2}\right)$ at $S_{1}=+2$, where it is pinched to a point again. The piece labelled by norm 3 is symmetric; it extends from $\left(-\frac{1}{2},-\frac{1}{2}\right)$ at $S_{1}=-2$ through $x_{3^{\prime}}$ or $\left(-1,-\frac{2}{3}\right)$ 

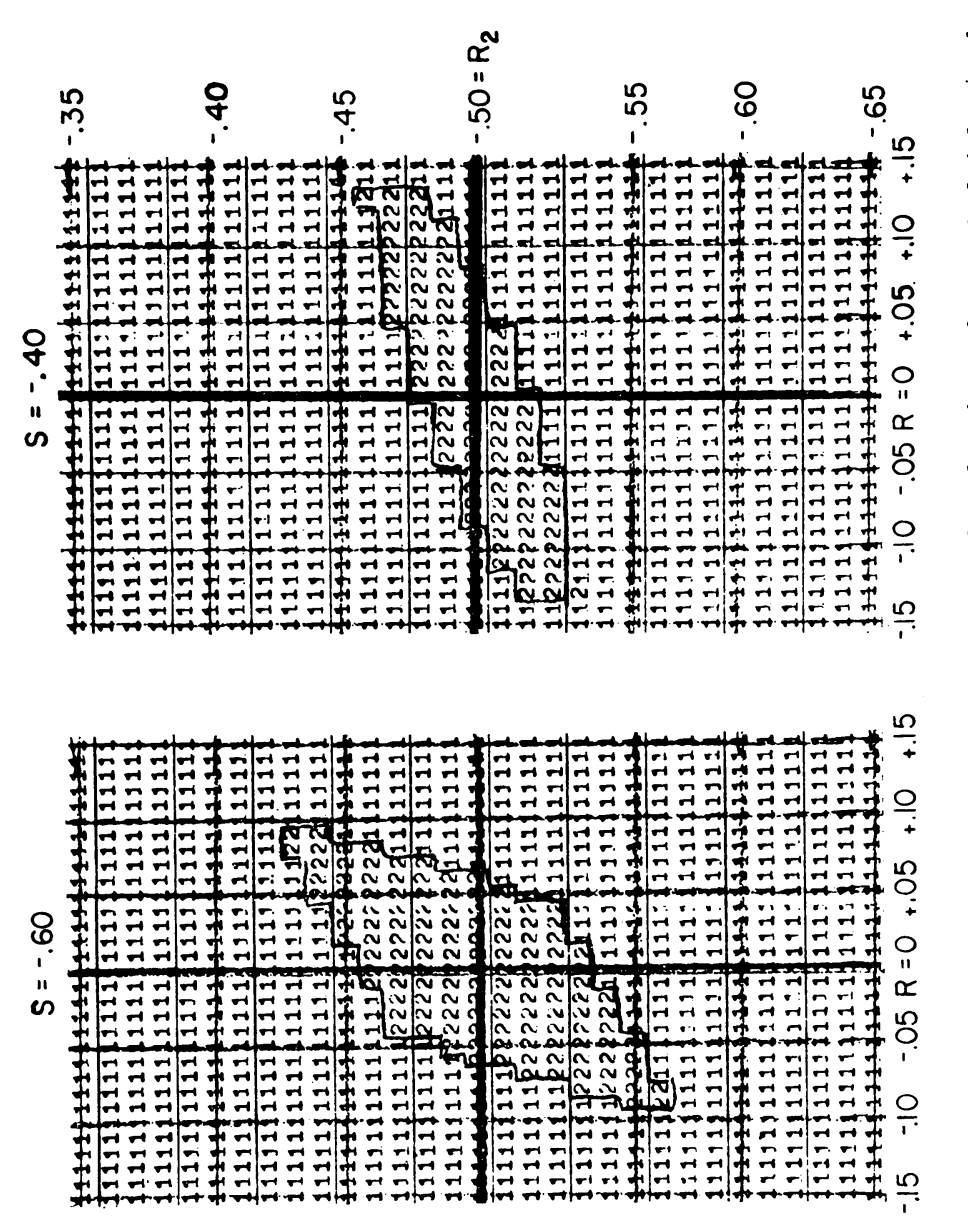

$\overrightarrow{2}$

宾

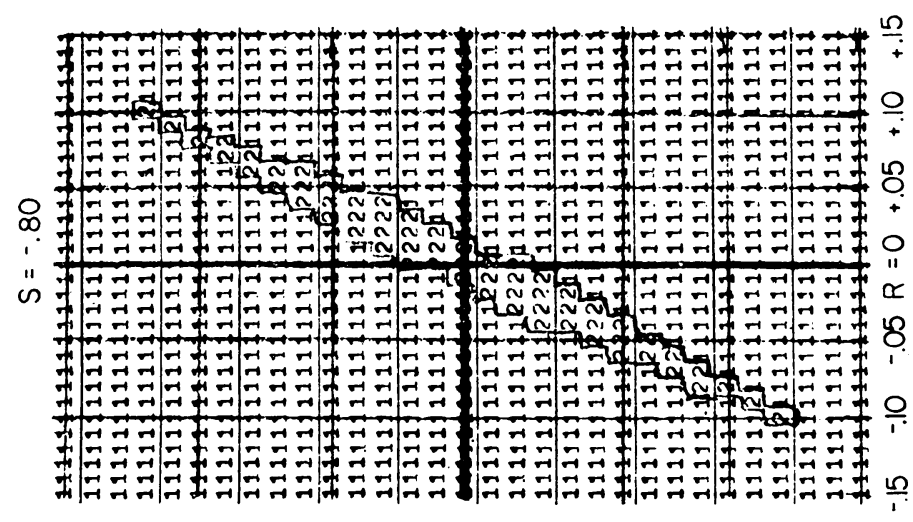



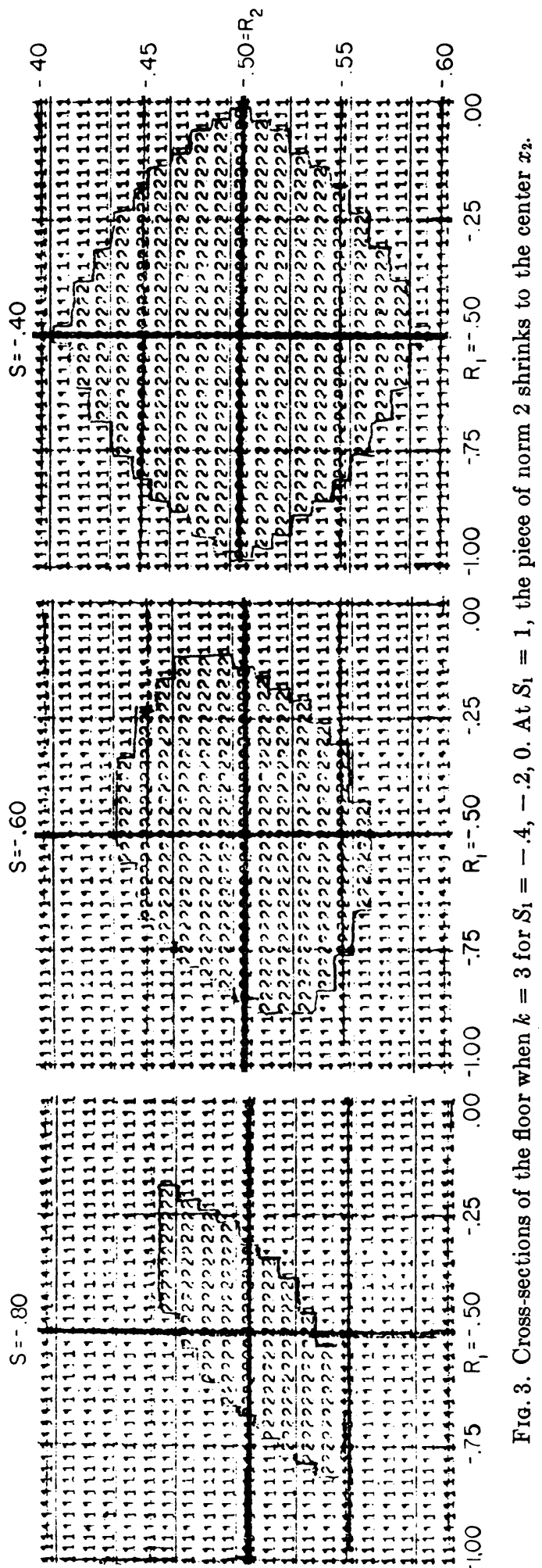
$\equiv\left(0,-\frac{2}{3}\right)$ at $S_{1}=0$ and then from $\left(0,-\frac{2}{3}\right)$ at $S_{1}=0$ back to $\left(-\frac{1}{2},-\frac{1}{2}\right)$ at $S_{1}$ $=+2$. (See Remark (g) in Section 13 below.)

The piece of norm 2 fits in more remarkably as shown in Fig. 4. It begins at $S_{1}=-2$ around its center $x_{2}$ represented twice or $\left(R_{1}, R_{2}\right)=\left(0,-\frac{1}{2}\right) \equiv\left(-1,-\frac{1}{2}\right)$ and expands so as to "swallow" all other norms by the time $S_{1}$ reaches 0 . Meanwhile, when $S_{1}$ reaches $S_{A}=-1.226 \cdots$ (approx.) the piece of norm 2 attaches itself to the pieces of norms 3 and 4 at point $Z_{A}$ where regions of norms $1,2,3$, and 4 all meet. (This is shown roughly in Fig. 4 by using $S_{1}=-1.2$.) By the time $S_{1}=-1$ then the piece of norm 2 splits the two pieces of norms 3 and 4 at a point $Z_{0}$ which is precisely the point at which we conjectured minimal height $S_{2}=.2$ in (4.2). As $S_{1}$ increases to 0 , the pieces of norms 3 and 4 separate and shrink to the centers $x_{3}$ and $x_{4}$ (see Table 4).

Of incidental interest is the fact that $Z_{A}$, the point of attachment just described (in Fig. 4) is not an intuitively obvious point. About a half-hour was devoted to running finer and finer grids in order to approximate it. The final grid used was at maximum accuracy permitted by an input of four decimal digits (a purely mechanical and unintentional limitation), namely

$$
\Delta R_{1}=\Delta R_{2}=\Delta S_{1}=.0001 \text {. }
$$

A good approximation seems to be

$$
\begin{array}{rlrl}
Z_{A} & =-1.5899+.8562 i, & Z_{\Delta}{ }^{\prime} & =.7695+.2851 i \\
\left(R_{1}, R_{2}\right) & =(-.4102,-.4816), \quad S_{1} & =-1.2256, \quad S_{2}=.2441
\end{array}
$$

but the exact values (possibly as surds) are elusive. Strangely enough, it is easy to get four conditions to determine $Z_{A}$ by examining optimizing denominators $\|\gamma Z+\delta\|=1$ for points $Z$ near $Z_{\Delta}$. We find four equations corresponding to the pairs

$$
(\gamma, \delta)=(1,-1),\left(2+6^{1 / 2}, 3+6^{1 / 2}\right),\left(3+6^{1 / 2}, 2+6^{1 / 2}\right),\left(2,1+6^{1 / 2}\right) .
$$

13. Concluding Remarks. (e) The combination $(\gamma, \delta)=(1, \delta)$ is so frequent that the program is made to have the additional convenience that such listings are omitted and when $\gamma=1$ the machine automatically ignores $\delta$ and comes up with $\alpha_{0}=0, \beta_{0}=-1$ when $\gamma=1$. This was done by a "dummy listing" in (10.2) consisting of

$$
1,0,0,0 ; \quad 0,0,-1,0 \text {. }
$$

Thus the table (10.2) is reasonably short consisting of one item (the "dummy") when $k=5$, seven items when $k=2$ or 3 , and 21 items when $k=6$. An error print-out informs us if some $(\gamma, \delta)$ had been inadvertently omitted from the table (except for $\gamma=1$ ).

(f) The machine is given a limit of 10 tallies (to an error print-out) for the iteration under discussion since a factor of $\epsilon_{+}{ }^{10}$ would be preposterously large. No error print-out was encountered in practice.

(g) Here it is clear why we need the matching of (9.1) and (9.3). The earlier program in [6] made it clear how to label each piece according to $|N(\gamma)|$ but we would not know how to match the various segments of norm 3 otherwise. 

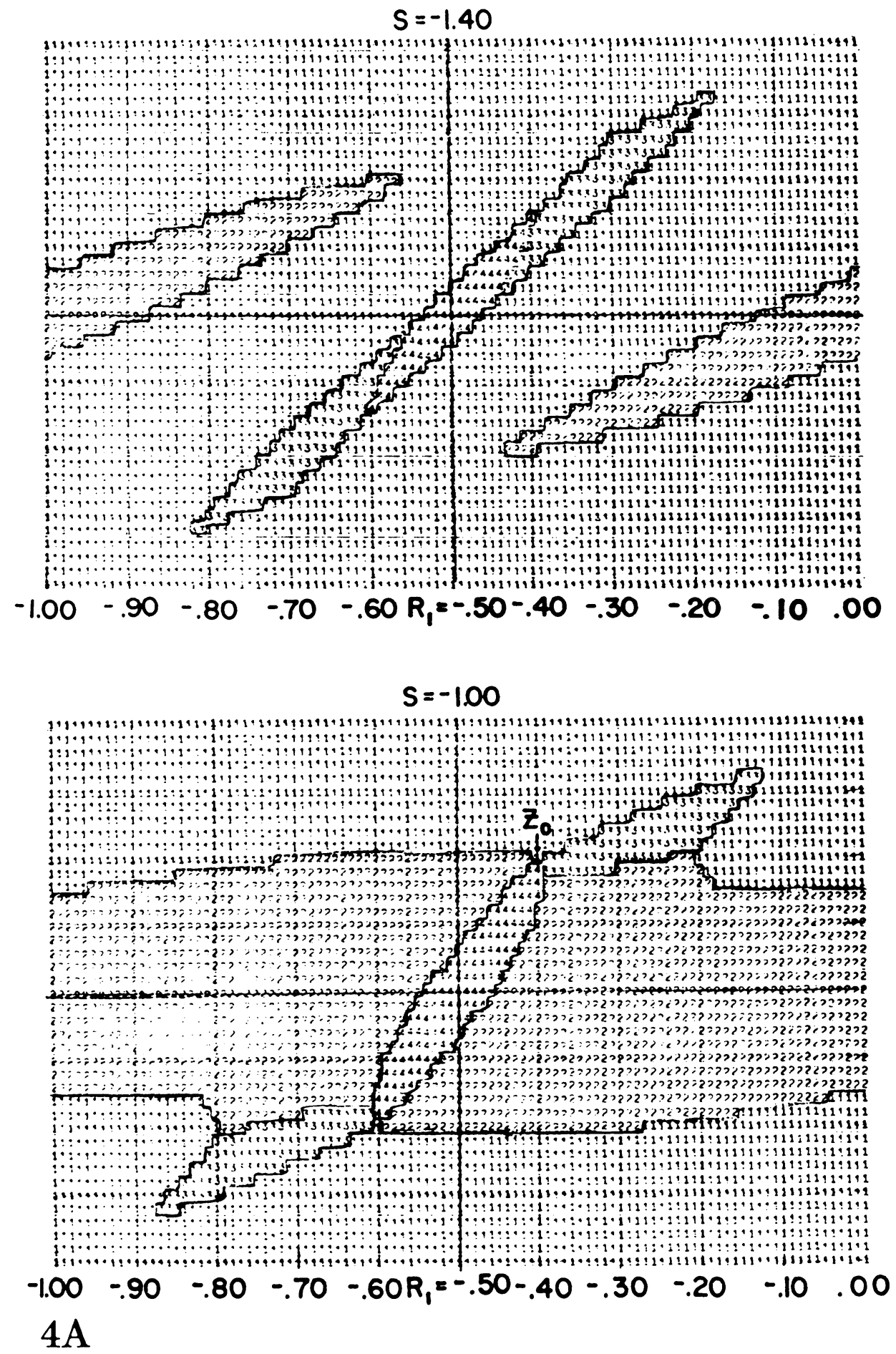

Fig. 4. Cross-sections of the floor when $k=6$ for $S_{1}=-1.4,-1.2,-1,-.6$. The piece of norm 3 is in the upper right of each cross-section while the piece of norm $3^{\prime}$ is in the lower left. The centers $x_{2}, x_{3}, x_{3^{\prime}}, x_{4}$ are the same on all cross-sections but are shown once only for simplicity. When $S_{1}=-2$, the pieces of norms $3,3^{\prime}, 4$ shrink to $x_{4}$ and the piece of norm 2 shrinks to $x_{2}$. When $S_{1}=0$, the pieces of norm $3,3^{\prime}$ and 4 shrink to $x_{3}, x_{3^{\prime}}$ and $x_{4}$ respectively while the piece of norm 2 spreads slightly further than shown in $S_{1}=-.6$. 

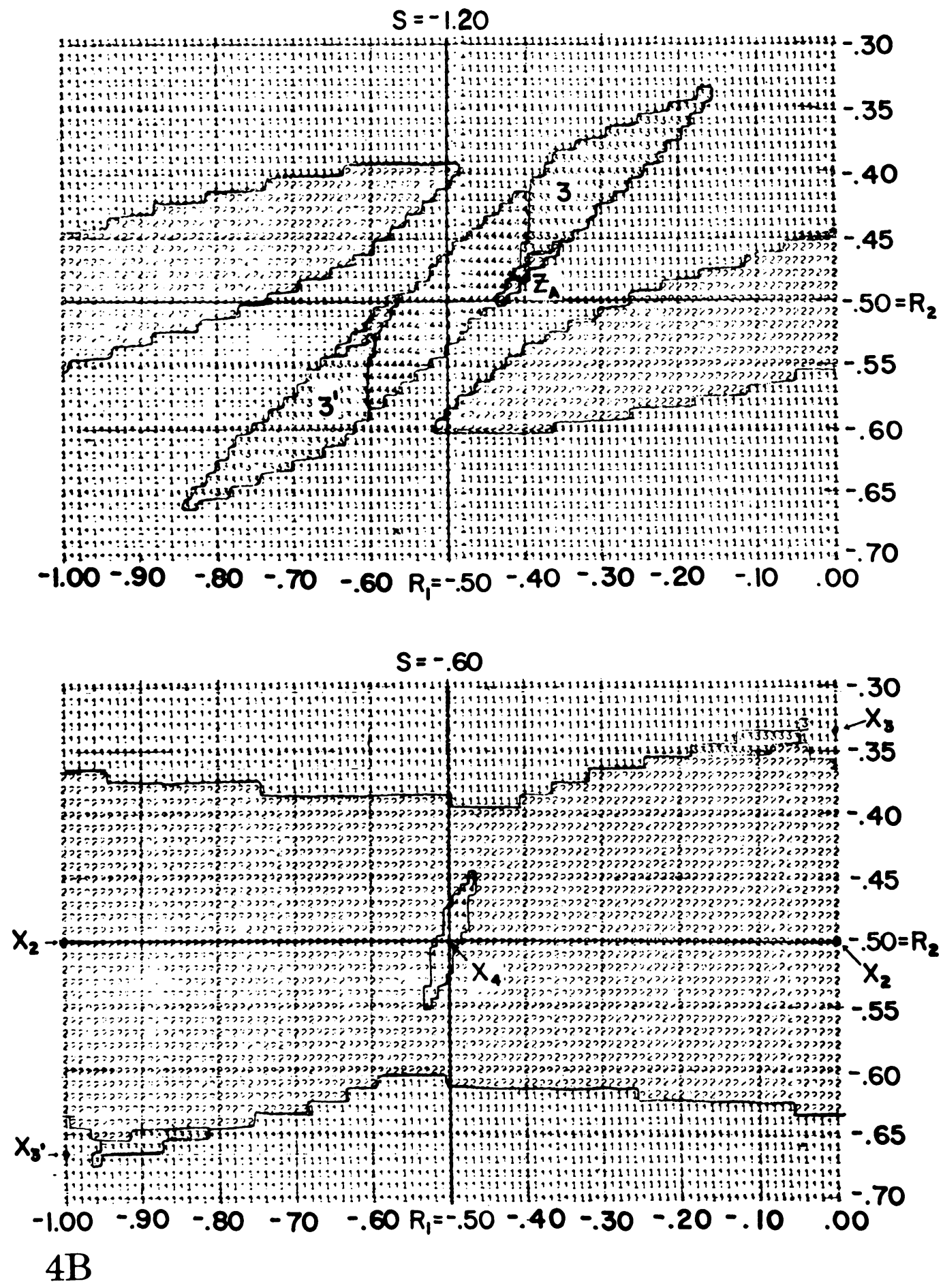
In summary, the modified program slowed down the process to the production of about 5 to 10 matching pairs (9.1) and (9.3) a second on the CDC 3600. Clearly the speed is far too slow for running the program directly on visual input-output facilities. The great aid achieved in visualizing four-dimensional regions cannot be denied if the time factor could be overcome. (Indeed, even the floors of threedimensional modular regions are extremely difficult to visualize, see [11].) Further work in visualization will undoubtedly require previous storage for fast access. We are still nowhere near some ultimate purpose such as "seeing" homology generators as immediately as in two-dimensional manifolds.

The University of Arizona

Tucson, Arizona

6. H. Corn, "A numerical survey of the floors of various Hilbert fundamental domains," Math. Comp., v. 19, 1965, pp. 594-605.

7. H. CoHN, "Note on how Hilbert modular domains become increasingly complicated," J. Math. Anal. Appl., vol. 15, 1966, 55-59.

8. K. B. GUNDLACH, "Die Fixepunkte einiger Hilbertscher Modulgruppen," Math. Ann., v. 157,1965 , pp. $369-390$.

9. M. HALL, Topology of Torus-Like Fibre Spaces, Ph.D. Dissertation, University of Arizona, Tucson, 1966.

10. N. STEEn Rod, The Topology of Fibre Bundles, Princeton Univ. Press, Princeton, N. J., 1951. MR 12, 522 .

11. W. WOODRUFF, Investigation of Congruence Subroups of Picard's Group with Respect to Topological Structure, Ph.D. Dissertation, University of Arizona, Tucson, 1967. 